\title{
A Novel Approach to Learning Models on EEG data Using Graph Theory features - A Comparative Study
}

\author{
Bhargav Prakash ${ }^{1, \ddagger}$, Gautam Kumar Baboo ${ }^{1, t, \neq}$ and Veeky Baths ${ }^{1, \ddagger_{*}}$ \\ ${ }^{1}$ Cognitive Neuroscience Lab, Department of Biological Sciences; f20170157@goa.bits-pilani.ac.in \\ ${ }^{1}$ Cognitive Neuroscience Lab, Department of Biological Sciences; p20130404@goa.bits-pilani.ac.in \\ ${ }^{1}$ Cognitive Neuroscience Lab, Department of Biological Sciences; veeky@goa.bits-pilani.ac.in \\ * Correspondence: veeky@goa.bits-pilani.ac.in; Tel.: +91 8322580436 \\ $\ddagger$ These authors contributed equally to this work.
}

\begin{abstract}
Functional Connectivity analysis using Electroencephalography signals is common. The EEG signals are converted to networks by transforming the signals into a correlation matrix sand analyzing the resulting networks. Here, four learning models, namely, Logistic Regression, Random Forest, Support Vector Machine, and Recurrent Neural Networks, are implemented on two different types of correlation matrices: static and dynamic (time-resolved), to classify them

${ }_{6}$ either on their psychometric assessment or the effect of therapy; These correlation matrices are different from traditional learning techniques in the sense that they incorporate theory-based graph features into the learning models, thus providing novelty to this study. The EEG data used in this study is trail-based/event-related. The classifications based on RNN provided higher accuracy $(74-88 \%)$ than the other three models( $50-78 \%$ ). Instead of using individual graph features, 11 a correlation matrix provides an initial test of the data. When compared with the time-resolved 12 correlation matrix, it offered a 4-5\% higher accuracy. The time-resolved correlation matrix is better 13 suited for dynamic studies here; it provides lower accuracy when compared to the correlation 14 matrix, a static feature.
\end{abstract}

Keywords: EEG; Emotional States; Working Memory; Depression; Anxiety; Graph Theory; Classi fication; Machine Learning; Neural Networks.

\begin{abstract}
171. Introduction
${ }_{18}$ Electroencephalography $(E E G)$ is a commonly used neuroimaging tool. Its applica ${ }_{19}$ tion ranges from; clinical capacity such as sleep disorder studies, seizure detection to 20 commercial circumstances such as EEG-controlled games[1].The EEG data is a matrix, ${ }_{21}$ which consists of electric potentials. This form of EEG data makes it easy to use machine ${ }_{22}$ learning models[2]. With its high temporal resolution, EEG data can provide information ${ }_{23}$ regarding the functional connectivity within the brain, thereby providing a topological ${ }_{24}$ understanding of the functioning of the human brain[3].This is usually carried out by ${ }_{25}$ transforming the electrical potentials into a correlation matrix[4].
\end{abstract}

To understand the functional aspects of the brain under conditions of executive ${ }_{27}$ functions and emotional states viz. depressive or anxious, it is vital to study them in 28 terms of networks and the best way to do it, but with the help of EEG signals. At ${ }_{29}$ present learning, models use either the properties of the EEG signal such as amplitude, ${ }_{30}$ frequency, or event-related potentials as features or graph properties such as centrality measures which are nodal metrics, or edge metrics such as shortest path length.

Network analysis and Learning models on neuroimaging data have enabled re searchers to study the human brain's functional and structural connectivity [5].Here, graph metrics are used as features for a deep learning model, apart from the standard spectral and temporal characteristics that are traditionally used[6].Different static and dynamic features are studied to understand which features are best suited for visual 
working memory tasks[7]Both CNN and RNN are tested and validated for their performance on the datasets.

Previous work on emotional states such as depression and anxiety in the space of EEG and machine learning was carried out using signal features such as power or frequency bands[8].Learning models such as probabilistic, nearest neighbor, neural network, and tree-based have been implemented on DASS scores, here the random forest model provided accuracy in classification of three states, i.e., depressive, anxious or stressed at $84 \%, 85 \%$, and $84 \%[9,10]$.

A study, clinically depressed patients and normal controls with the implementation of learning models on EEG signals using features such as frequency bands and non linear features such as detrended fluctuation analysis (DFA), Higuchi fractal, correlation dimension, and Lyapunov exponent provided an $83.3 \%$ accuracy while using logistic regression[11]. Similarly, visual and verbal working memory studies using EEG have been carried out using event-related potentials(ERP's) and the subsequent construction of functional connectivity of these ERPs[5]. Study using EEG and deep learning models involves EEG signals broken into smaller windows for training and testing[12].The high temporal resolution being the nature of EEG signals adds an additional step into curating these smaller datasets for analysis. This step can induce a bias based on cognitive noise between participants. An SVM implementation to classify Schizophrenic patients and healthy controls based on working memory task yield an accuracy of $>74 \%[13]$.

Learning models on EEG data recorded during visual short-term memory task included SVM and Random forest, which used raw EEG signals and the psychometric assessment scores and reaction times which provided an accuracy of approximately $90 \%[14]$ Other implementations of SVM using frequency bands as features on similar psychological tests yield a 98\% accuracy[15]While using ERPs in the time domain, power ${ }_{62}$ spectra and eye-tracking as features provided accuracy in the range of $40 \%$ to $60 \%[7]$.

${ }_{63}$ Given that the intermediate step between EEG signal analysis and functional con nectivity analysis is the use of a correlation matrix; In this study, we explore the utility 65 of the same along with a time-resolved correlation matrix for a comparative learning model study. The comparison of the two correlation matrices can shed some light on ${ }_{67}$ the nature of EEG activity in these emotional and cognitive states. Here the EEG data ${ }_{68}$ for working memory and emotional states are used from a total of 359(25(DASS21), ${ }_{69} 122$ (Selection Task), 29(WM-Lab), 27(Visual-WM+drug) \& 156(Verbal-WM))participants. 70 Both EEG data and associated psychometric assessment scores are used for the learning ${ }_{71}$ model study. Two high-accuracy models, i.e., recurrent neural network and Random ${ }_{72}$ forest, belong to the neural networks method and ensemble method. And two high ${ }_{73}$ interpretable models, a kernel-based method- Support Vector Machine and the Logistic ${ }_{74}$ Regression model, are examined and compared.

\section{Results}

The performance of RNN classifiers shows up to $94.50 \%$ and $88.64 \%$ accuracy for each of the working memory tasks, which outperforms most of the previous works reviewed. The performance of R.F. and L.R. classifiers are relatively sub-par compared to RNNs but still comparable to previously obtained results. The poor performance of SVMs highlights the shortcomings of the method adopted in this study in algorithms that are sensitive to the dimensions of the data. The impressive performance of RNNs can be attributed to their innate ability to extract correlated features, which are not visible in traditional statistical methods, within the data with the help of their stacked networks and activation functions. The standard performance of R.F. and L.R. algorithms highlights the validity of the method adopted in this study and the enormous scope it provides for further improvement.

Further the data from the public database provides higher accuracy in all four models when compared to the in-house data. On an average there seems to be a difference of $40-60 \%$ accuracy between the two groups. 


\subsection{Figures, Tables and Schemes}

\begin{tabular}{|c|c|c|c|c|c|c|c|c|}
\hline$\omega$ & beveren & nover & Sencimen & chen & Nincelevers: & 0 & $\wedge$ & $s$ \\
\hline 1 & 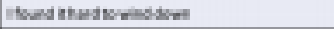 & a & 2 & $i$ & 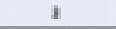 & & & \\
\hline 1 & 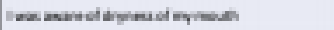 & a & a & 2 & 1 & & & \\
\hline 3 & 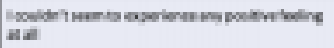 & 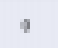 & 1 & 2 & 3 & & & \\
\hline 4 & 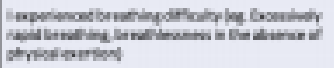 & a & $=$ & 2 & $\boldsymbol{x}$ & & & \\
\hline$s$ & 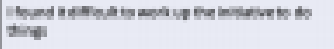 & a & a & 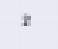 & 1 & & & \\
\hline • & 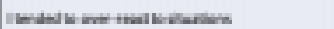 & a & $\mathrm{a}$ & $z$ & $\boldsymbol{x}$ & & & \\
\hline$x$ & 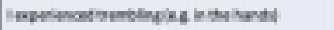 & a & a & 2 & 3 & & & \\
\hline$\approx$ & 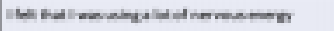 & a & $=$ & 2 & 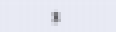 & & & \\
\hline$\bullet$ & 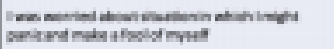 & a & $=$ & $z$ & $x$ & & & \\
\hline $\mathbf{\infty}$ & 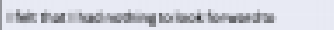 & a & $=$ & $i$ & $\boldsymbol{x}$ & & & \\
\hline st & 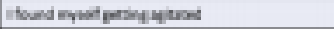 & $a$ & $\mathrm{a}$ & $i$ & $x$ & & & \\
\hline ม & 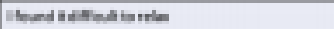 & a & 8 & 2 & s & & & \\
\hline at & 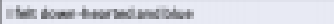 & a & a & 2 & $\boldsymbol{x}$ & & & \\
\hline $\boldsymbol{u}$ & 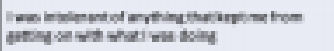 & a & 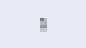 & $z$ & 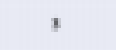 & & & \\
\hline Is & 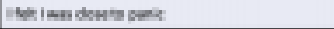 & 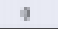 & 1 & 2 & 3 & & & \\
\hline * & 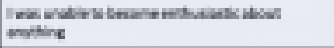 & 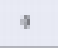 & I & 2 & 3 & & & \\
\hline$m$ & 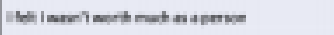 & a & $s$ & ? & , & & & \\
\hline at & 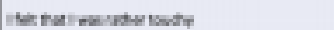 & a & $=$ & i & , & & & \\
\hline * & 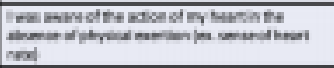 & a & $\mathrm{a}$ & 2 & $x$ & & & \\
\hline 30 & 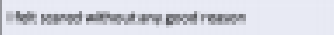 & $\rightarrow$ & 1 & 2 & 3 & & & \\
\hline $\mathbf{n}$ & 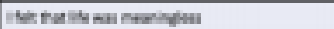 & $a$ & $\mathrm{~s}$ & $x$ & 3 & & & \\
\hline & & & & & $\operatorname{Ten}$ & & & \\
\hline
\end{tabular}

Figure 1. DASS 21 questionnaire example.

Table 1: Classifying Emotional States from the DASS 21 Data

\begin{tabular}{|l|c|c|c|c|}
\hline Emotional State/ & $\begin{array}{c}\text { Logistic } \\
\text { regression }\end{array}$ & $\begin{array}{c}\text { Random } \\
\text { Forest }\end{array}$ & SVM & RNN \\
\hline Depression & $35.06 \%$ & $28.60 \%$ & $\begin{array}{c}27.60 \\
\%\end{array}$ & $\begin{array}{c}34.75 \\
\%\end{array}$ \\
\hline Anxiety & $28.40 \%$ & $34.45 \%$ & $\begin{array}{c}30.85 \\
\%\end{array}$ & $\begin{array}{c}38.85 \\
\%\end{array}$ \\
\hline Stress & $31.10 \%$ & $33.20 \%$ & $\begin{array}{c}31.70 \\
\%\end{array}$ & $\begin{array}{c}36.40 \\
\%\end{array}$ \\
\hline
\end{tabular}



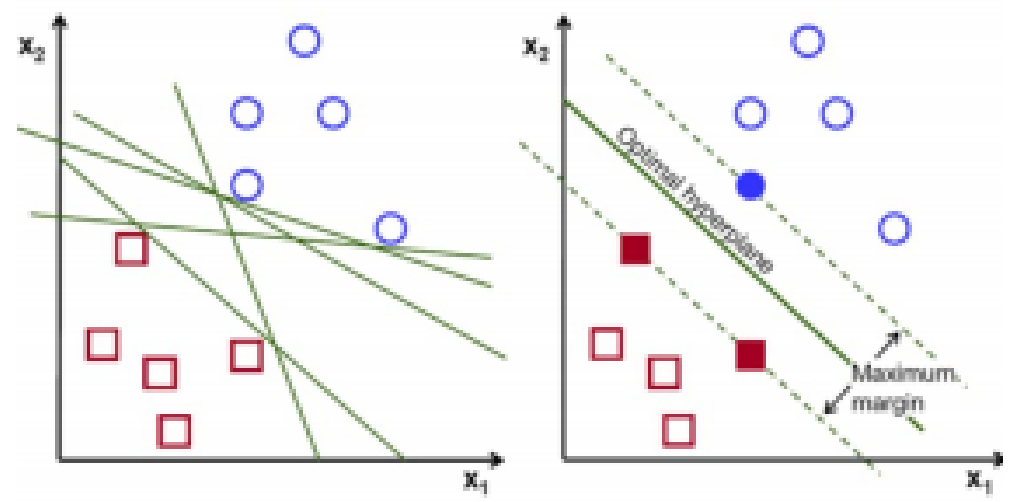

Figure 2. Support Vector Machine

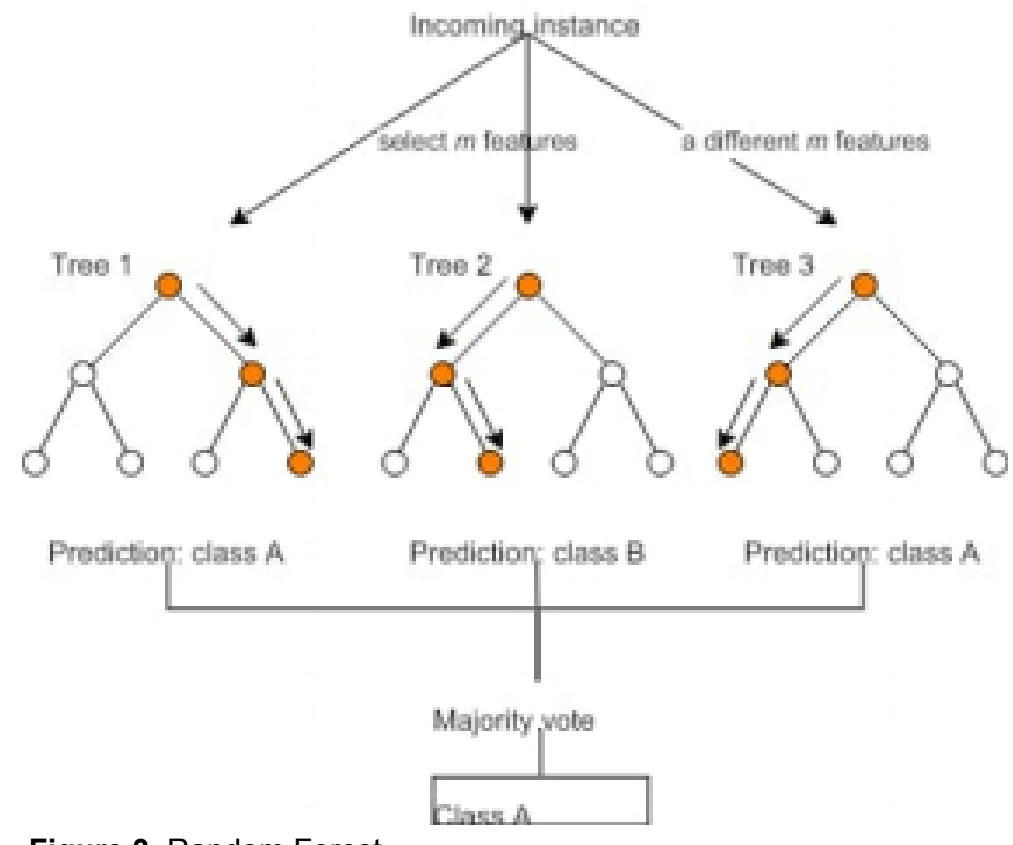

Figure 3. Random Forest

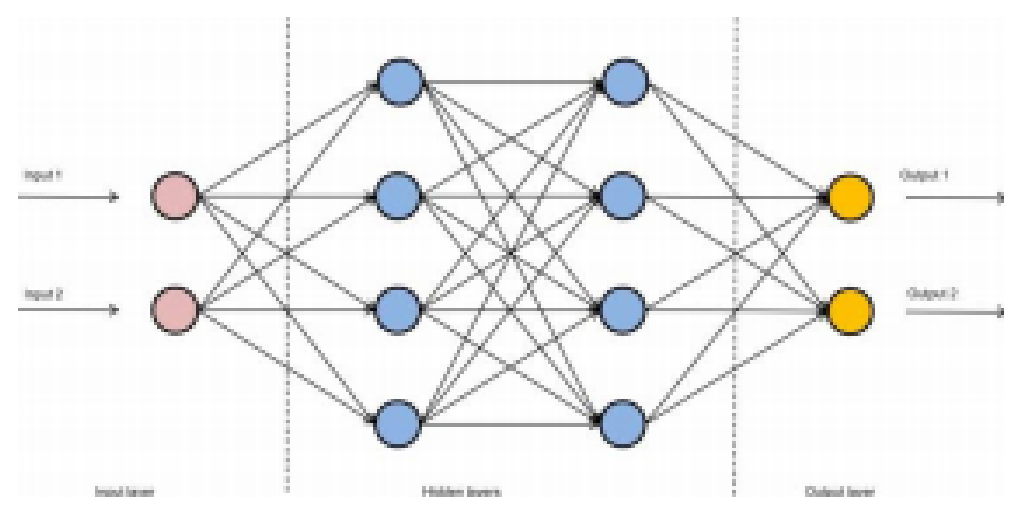

Figure 4. Recurrent Neural Network

Table 2: Accuracy of Classifying Emotional States from the Probabilistic Selection Task Data

\begin{tabular}{|l|c|c|c|c|}
\hline $\begin{array}{c}\text { Emotional State/ } \\
\text { Learning Model Accuracy }\end{array}$ & $\begin{array}{c}\text { Logistic } \\
\text { Regressio } \\
\mathrm{n}\end{array}$ & $\begin{array}{c}\text { Random } \\
\text { Forest }\end{array}$ & SVM & RNN \\
\hline Depression & $71.33 \%$ & $73.46 \%$ & $\begin{array}{c}61.78 \\
\%\end{array}$ & $\begin{array}{c}88.64 \\
\%\end{array}$ \\
\hline Anxiety & $64.56 \%$ & $78.66 \%$ & $\begin{array}{c}65.27 \\
\%\end{array}$ & $80.75 \%$ \\
\hline
\end{tabular}




\section{Discussion}

Implementation of learning models on imaging data to study emotional states provided reliable results in the past[16]. With the use of both high accuracy(RNN and R.F.) and high interpretability (SVM and L.R. model), we can look for nonlinear relationships, non-smooth relationships, along well-defined relationships. The present study demonstrated that a correlation matrix can be used in learning models and provides good accuracy. Further yielded higher accuracy rates with well-structured data obtained in a controlled environment, as were the working memory tasks, indicating superior discriminatory performances when assessing mental tasks. In addition, the present study is discriminatory towards poorly collected and insufficient data.

${ }_{101}$ From running the classification models on both types of datasets: correlation and 102 time-resolved correlation matrices, we find that the two classification models: Random 103 Forest Classifier and RNN classifier perform relatively better when the correlation is ${ }_{104}$ not time-resolved. The performance dips across both the Verbal Memory and Working 105 memory datasets for time-resolved correlation. This provides scope for further research 106 as to why dynamic methods may not be a better fit for Neural Networks and Decision 107 trees based classification models.

The engagement of participants in the five experimental paradigms is by nature dynamic states of EEG activity. With this in mind, the use of a time-resolved correlation matrix is explored alongside the correlation matrix, both features of the time domain of the signal. Since the results indicate the use of the static feature is most suitable for such cognitive states, it sets the stage to explore the other static and dynamic features of trail-related EEG data. At this stage, this investigation sets the stage for exploring the possibility of using these features as markers for the cognitive footprints of psychopathologies such as memory and emotional state deficits.

The results indicate that using graph metric for static features is optimum. Using computerized administration of the test's rules out pressure to perform or be dishon est. This study compares learning models on similar paradigm EEG data helps with functional connectivity study.

\subsubsection{Limitations}

Although RNN and random forest models providing high accuracy, both these ${ }_{122}$ methods have longer run times when compared to the other two. In the current study, ${ }_{123}$ the lack of defined healthy control groups across the datasets can be addressed, which ${ }_{124}$ can help improve the accuracy of the models. This imbalance can be addressed using ${ }_{125}$ larger data and a robust learning model[17]. Single trials in the case of in-house data ${ }_{126}$ set and using DASS 21 for the first time as a computerized test and EEG could explain ${ }_{127}$ the lower accuracy across the models associated with this data. This also applies to the 128 visual working memory data recorded in the lab. Using graph features on the EEG data ${ }_{129}$ is time-consuming because graph features can range from nodal metrics to local/global ${ }_{130}$ network characteristics that need to be considered features. Simultaneously cheery ${ }_{131}$ picking graph metric(s) can introduce a bias that has to be considered into the study and ${ }_{132}$ address at a later point with defined statistical analysis.

\section{Materials and Methods}

\subsection{Data sets}

In this study, five EEG data sets are used, of which two were recorded in-house and three are from a public database. Among the two recorded in-house, 25 participants are from Sternberg Visual Working Memory Task, and 29 participants are from the DASS 21 questionnaire (approved by the Institute Research Ethics Committee (IHEC-40/16-1)) using a 32 Channel EGI geodesic system(appendix figure A1.). From the OpenNeuro dataset, 122 participants from Probabilistic Selection Task(OpenNeuro Dataset Accession Number: ds003474) is recorded using a 64 channel Synamps system, 156 participants from Verbal working memory Task(OpenNeuro Dataset Accession Number: ds003565) is 
recorded using a 19 channel 10-20 system Mitsar-EEG-202 amplifier, and 27 participants from visual working memory task(OpenNeuro Dataset Accession Number: ds003519) are used. A total of 359 participants' EEG data is used here.

DASS 21 questionnaire is a 21 item self-administered test; this test contains seven sets of questions to assess the three emotional states; depressive, anxious, and stressed. A participant responds with a score ranging from 0 to 3 , with 0 meaning never and three meanings almost always. Scores for each category are cumulative; a rating between normal to severe is provided at the end of the test. These scores are then used in ${ }_{151}$ classifying the participants for the training dataset(See figure 1.).

The task, Probabilistic Selection and Depression(public database), has two tests, the Becks Depression Inventory and the State-Trait Anxiety Inventory[18]. The scores of these tests again range from normal to severe. For the Probabilistic Selection Task[19]the 155 participants were administered the Beck Depression Inventory(BDI) and State-Trait AnX ${ }_{156}$ iety Inventory(STAI). Here, BDI scores that are lesser than or equal to 19 are considered ${ }_{157}$ zero, and greater than or equal to 20 as one; likewise, for STAI scores, equal to and lesser ${ }_{158}$ than 55 , are considered as zero and greater than or equal to 56 as one.

159 Visual working memory(in-house recording) is a modified Sternberg working ${ }_{160}$ memory task(Designs, 2021), which involves a visual chart that needs to be memo ${ }_{161}$ rized/committed to memory, followed by tasks to complete based on the recollection of ${ }_{162}$ the chart from memory.

${ }_{163}$ Visual Working Memory + Cabergoline(1.25 mg) Challenge(public database), here ${ }_{164} \mathrm{a}$ drug that can improve memory functions and placebo is administered to a small group 165 of participants. The placebo and drug groups are used for classification. For the Visual ${ }_{166}$ Working Memory+Cabergoline[20]challenge data, two sessions are carried out for each ${ }_{167}$ participant, one with a placebo and the other with the drug. Here the placebo is treated ${ }_{168}$ as zero and drug administered session as one[21].

${ }_{169}$ Finally, Verbal working memory(public database)[22] consists of the EEG recorded 170 in a modified Sternberg working memory paradigm with two types of tasks: with mental 171 manipulations (alphabetization) and simple retention (TASK) and three levels of load: 5, 172 6 , or 7 letters to memorize (LOAD). Apart from exploring the utility of the correlation ${ }_{173}$ matrix, a comparison between the data recorded in-house and the public database is 174 carried out using the accuracy of the models.

\section{4.2. Methods}

176 4.2.1. Data Processing

${ }_{177}$ Given the sensitivity of the EEG signals, it is imperative to preprocess them before ${ }_{178}$ any other analysis of the data is carried out. Therefore; the EEG data is filtered to remove ${ }_{179}$ line noise $(50 \mathrm{~Hz})$, bandpass filters, removal of bad channels, and artifact removal, and ${ }_{180}$ this data is converted into a correlation matrix and time resolved correlation matrix; 181 these operations are carried out on the Brainstorm package[23]on MATLAB.

\subsection{Learning Models}

After preprocessing and feature extraction of the original EEG data, the correlation matrix is used as input to different classifiers, including traditional machine learning algorithms and neural networks tuned in line with our data. The models used are Logistic Regression, Random Forest, Support Vector Machine, and Recurrent Neural Networks(RNN) to classify the EEG data. The performance evaluation of the different classifiers is examined using a confusion matrix, whose components are T.P., TN, F.P., F.N. Further, the accuracies are calculated using these measures, using the formula:

$$
\text { Accuracy }=(T P+T N) /(T P+F P+T N+F N) * 100
$$

T.P.: True Positives T.N.: True Negatives F.P.: False Positives F.N.:False Negatives. 
${ }_{185}$ A Logistic Regression model with Gaussian Kernel and Laplacian Prior is used for ${ }_{186}$ classification. The Gaussian kernel optimizes the separation between data points in the ${ }_{187}$ transformed space obtained in preprocessing, while the Laplacian Prior enhances the ${ }_{188}$ sparseness of learned L.R. regressors to avoid overfitting(Wu et al., 2018). A multinomial 189 L.R. model where the probability that an input feature xi belongs to class $k$ is given by:

190 : feature vector

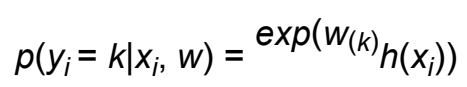

$$
\sum_{k=1}^{K} \exp \left(w^{(k)} h\left(w_{i}\right)\right.
$$

$$
\begin{aligned}
& { }_{191} k \text { : class } \\
& \qquad h\left(x_{i}\right) \text { : linear transformation function of } x_{i} \\
& { }_{192}^{193} W \text { : logistic regressors }
\end{aligned}
$$

\section{4.3.2. Support Vector Machine (SVM)}

${ }_{195}$ Apart from the application of SVM on EEG data, implementation of SVM on ${ }_{196}$ MRI data to classify between major depressive disorder and bipolar disorder provided 197 accuracy upto $45 \%$ to $90 \%$ [24]. The main reason behind using SVM is to leverage its 198 relatively less computational power to produce a significant accuracy and to reduce 199 possible redundant information (which is very common in EEG datasets) residing in 200 the data. The input data is mapped to a higher dimensional vector space using a linear ${ }_{201}$ kernel function to find a hyperplane for classification.

$$
w * z-b=0 \text { (3) }
$$

202 W: normal vector

${ }_{203} \mathrm{~b}$ : bias of separation of hyperplane

\section{4.3.3. Random Forest (RF)}

${ }_{205}$ A Random Forest classifier that uses an ensemble learning approach towards 200 prediction is used. R.F. classifier works in a similar way as the decision tree classifier, 207 only with an ensemble learning approach added to it. The first step is the creation of ${ }_{208}$ many random decision trees, each predicting a particular class according to the features 209 given to it. Once each tree predicts a class, voting is carried out to take into consideration 210 the final class according to a majority. The output is then the class that has the majority ${ }_{211}$ voting.

\subsubsection{Recurrent Neural Network (RNN)}

Previous work on the implementation of neural networks on EEG signals has been fruitful, which provided accuracy in the range of $81 \%$ to $94 \%[25]$. RNN was a good model for studying both working memory[26,27] and emotional state[28] EEG data when compared to other models such as SVM or deep belief networks[29]on that note the following RNN model is implemented. The RNN is implemented through a Long Short Term Memory (LSTM) model[6,30], producing exemplary results on sequential data, such as EEG data. A sequential model is used to build the LSTM, which is a linear stack of layers. The first layer is an LSTM layer with 256 memory units, and it defines the input shape. This is done to ensure that the next LSTM layer receives sequences and not just randomly scattered data. The next layer is a Dense layer with a 'sigmoid' activation function. A dropout layer is applied after each LSTM layer to avoid over-fitting of the model. The model is then trained and monitored for validation accuracy using loss as 'binary cross-entropy, optimizer as 'adam' and metrics as 'accuracy.'

$$
H(q)=-1 / N \sum_{i}^{y} * \log \left(p\left(y_{i}\right)\right)+\left(1-y_{i}\right) * \log \left(1-p\left(y_{i}\right)\right)
$$


$H(q)$ : binary cross entropy

$p\left(y_{i}\right)$ : probability of belonging to class $y_{i}$

\section{Conclusions}

The time-series nature of the EEG data, which is an effective form of neuroimaging data. Using such EEG datasets recorded on the working memory and emotional state assessment paradigms, The Previous work on implementing learning models on EEG data consists of using features from the signal processing field. These studies provide an insight into the possible electrical activity of each lobe(s) associated with the behavior. However, they fall short while explaining the possible functional connectivity between the regions of the brain. The application of the correlation matrix can be implemented as a first step into choosing the appropriate learning model for studying the emotional or working memory EEG data. This study reveals that using a correlation matrix instead of a time-resolved correlation matrix even under trail-based EEG data is a better-suited input for learning models when compared to a dynamic feature such as the time-resolved correlation matrix. This brings us to the experiments themselves. The memory tasks and psychometric assessment tests-BDI, STAI and DASS 21, involve different brain regions, given that they have to be functionally connected to respond to the questions in these tests. This study provides a basis for studying the cognitive footprints for memory deficits, depression, anxiety and stress. Further, it is observed that RNN performs the best compared with the other three models implemented in this study.

${ }_{233}$ Author Contributions: Conceptualization, Gautam Kumar and Veeky Baths; methodology, Bhar ${ }_{234}$ gav Prakash; software, Bhargav Prakash; validation, Gautam Kumar and Bhargav Prakash; formal 235 analysis, Gautam Kumar and Bhargav Prakash.; investigation, Gautam Kumar and Bhargav; 236 resources, Veeky Baths.; data curation, X.X.; writing—original draft preparation, Gautam Kumar ${ }_{237} 2$ and Bhargav Prakash; writing-review and editing, Veeky Baths; visualization, Bhargav Prakash 238 and Gautam Kumar; supervision, Veeky Baths; project administration, Veeky Baths; funding 239 acquisition, Veeky Baths. All authors have read and agreed to the published version of the 240 manuscript.

${ }_{241}$ Funding: We thank the Department of Science and Technology, Government of India for the 242 grant(SR/CSRI/50/2014(G))and Department of Biological Sciences, BITS, Pilani -K.K. Birla Goa ${ }_{243}$ Campus for the infrastructure support.

244 Institutional Review Board Statement: The study was conducted according to the guidelines of 245 the Declaration of Helsinki, and approved by the Institutional Ethics Committee of Birla Institute 246 of Technology and Science, Pilani (IHEC-40/16-1).

${ }_{247}$ Informed Consent Statement: Informed consent was obtained from all participants involved in 248 the study. Written informed consent has been obtained from the participants to publish this paper.

${ }_{249}$ Data Availability Statement: In-house data shall be provided on request.

${ }_{250}$ Acknowledgments: We thank the Department of Science and Technology, Government of India ${ }_{251}$ for the grant(SR/CSRI/50/2014(G))and Department of Biological Sciences, BITS, Pilani -K.K. Birla ${ }_{252}$ Goa Campus for the infrastructure support.

${ }_{253}$ Conflicts of Interest: Declare conflicts of interest or state "The authors declare no conflict of 254 interest.

\section{${ }_{255}$ Appendix A}

${ }_{256}$ Appendix A. 1 


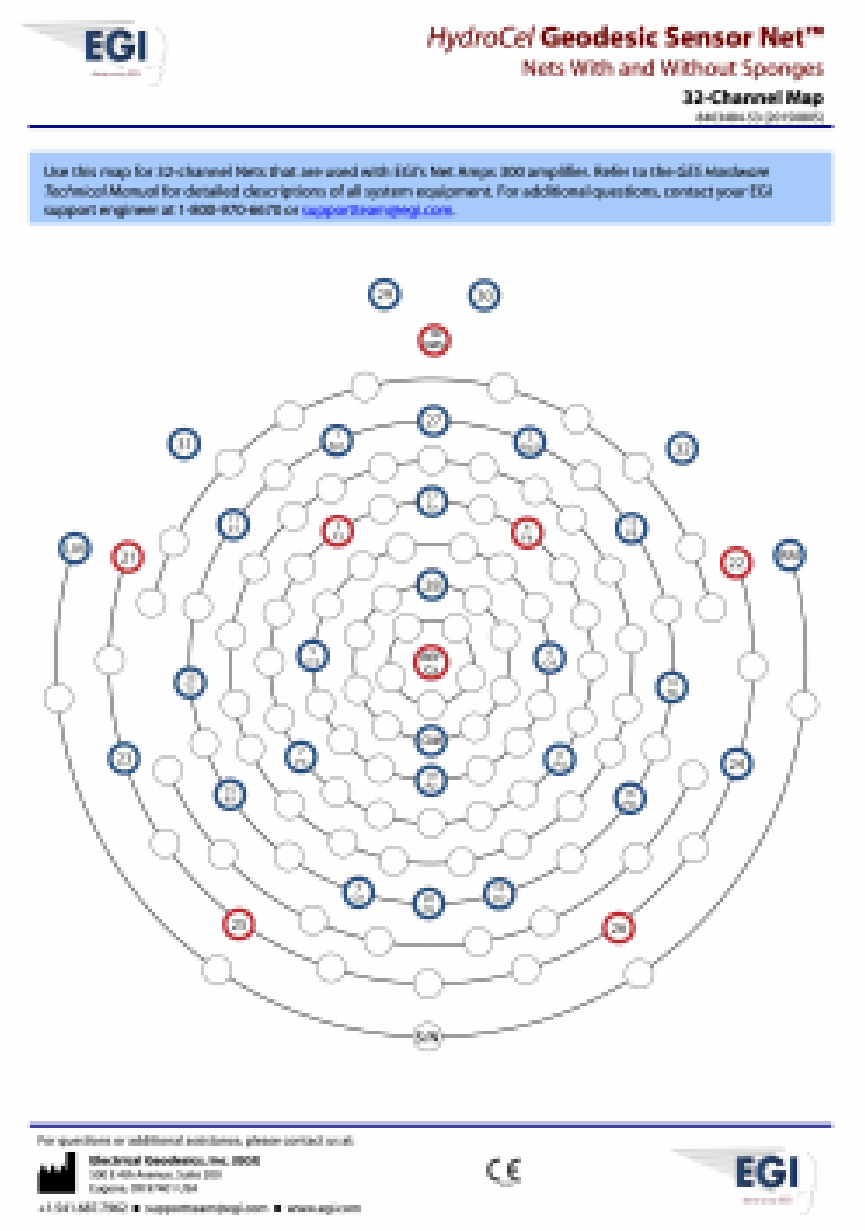

Figure A1. EEG Sensor Placement.

Table 3: Accuracy of Classifying Placebo vs Drug induced Memory Task conditions

\begin{tabular}{|l|c|c|c|c|}
\hline Condition & $\begin{array}{c}\text { Logistic } \\
\text { regression } \\
(\% \\
\text { accuracy) }\end{array}$ & $\begin{array}{c}\text { Random } \\
\text { Forest }(\% \\
\text { accuracy) }\end{array}$ & $\begin{array}{c}\text { SVM } \\
(\% \\
\text { accuracy) }\end{array}$ & $\begin{array}{c}\text { RNN } \\
(\% \\
\text { accuracy) }\end{array}$ \\
\hline Placebo & 73.60 & 80.40 & 73.50 & 90.20 \\
\hline Drug & 71.80 & 81.60 & 76.80 & 92.80 \\
\hline
\end{tabular}


Table 4: Accuracy of Classifying Verbal Memory Task Conditions 5,6 or 7 letters

\begin{tabular}{|c|c|c|c|}
\hline & 5 & 6 & 7 \\
\hline$:$ & $\begin{array}{l}\text { Logistic } \\
\text { regression - } \\
\quad 66.66 \%\end{array}$ & $\begin{array}{l}\text { Logistic } \\
\text { regression - } \\
\quad 59.40 \%\end{array}$ & $\begin{array}{l}\text { Logistic } \\
\quad \text { regression - } \\
\quad 61.10 \%\end{array}$ \\
\hline$\vdots$ & $\begin{array}{l}\text { Random } \\
\text { forest }-65.50 \%\end{array}$ & $\begin{array}{l}\text { Random } \\
\text { forest }-69.40 \%\end{array}$ & $\begin{array}{l}\text { Random } \\
\text { forest }-76.70 \%\end{array}$ \\
\hline " & SVM - 60.15\% & SVM - 59.80\% & SVM - \\
\hline & RNN - 75.86\% & RNN - $70.40 \%$ & $54.70 .10 \%$ \\
\hline & & & RNN - \\
\hline & & & $71.50 \%$ \\
\hline$\vdots$ & $\begin{array}{l}\text { Logistic } \\
\text { regression - } \\
\quad 68.70 \%\end{array}$ & $\begin{array}{l}\text { Logistic } \\
\quad \text { regression - } \\
\quad 66.40 \%\end{array}$ & $\begin{array}{l}\text { Logistic } \\
\text { regression - } \\
\quad 63.40 \%\end{array}$ \\
\hline$\vdots$ & $\begin{array}{l}\text { Random } \\
\text { forest - } 70.60 \%\end{array}$ & $\begin{array}{l}\text { Random } \\
\text { forest }-65.80 \%\end{array}$ & $\begin{array}{l}\text { Random } \\
\text { forest }-68.30 \%\end{array}$ \\
\hline & SVM - 55.60\% & SVM - 50.20\% & SVM - 53.30\% \\
\hline & RNN - $74.80 \%$ & RNN $-70.60 \%$ & RNN - $79.60 \%$ \\
\hline
\end{tabular}

Table 5: Participants of Modified Sternberg Working Memory Task

$\begin{array}{llll} & \text { accuracy) } & \text { SVM } & \text { (\% accuracy) } \\ \text { Logistic } & \text { Random Forest } & \text { (\% accuracy) } & \\ \text { regression (\% } & \text { (\% accuracy) } & \text { RNN } & \end{array}$

Participant 0112.537 .528 .6012 .5 Participant 022528.3028 .6028 .60

Participant 0314.3037 .514 .3014 .30

Participant 045012.52525 Participant 0525252528.60 Participant 0625

12.512 .514 .30 Participant 0714.3042 .9012 .550 Participant 0812.52512 .5

12.5 Participant 095028.6022 .2225 Participant 10755014.6014 .60

Participant 1112.512 .528 .6022 .22 Participant 1237.55011 .1112 .5

Participant 1328.6014 .302528 .60 Participant 1412.512 .537 .514 .30

Participant 15252537.525 Participant 162512.512 .512 .5 Participant 17

28.60255033 .33 Participant 1812.537 .52514 .60 Participant 195012.537 .5

25 Participant 2014.3014 .3014 .3012 .5 Participant 212537.514 .3012 .5

Participant 2212.52522 .2214 .30 Participant 2314.302528 .6025 Participant

242512.512 .528 .60 Participant 255028.6012 .512 .5

\section{References}

1. Soufineyestani, M.; Dowling, D.; Khan, A. Electroencephalography (EEG) technology applications and available devices. Applied Sciences (Switzerland) 2020, 10, 1-23. doi:10.3390/app10217453.

2. Li, G.; Lee, C.H.; Jung, J.J.; Youn, Y.C.; Camacho, D. Deep learning for EEG data analytics: A survey. Concurrency Computation. John Wiley and Sons Ltd, 2019. doi:10.1002/cpe.5199.

3. Vecchio, F.; Miraglia, F.; Maria Rossini, P. Connectome: Graph theory application in functional brain network architecture. Clinical Neurophysiology Practice 2017, 2, 206-213. doi:10.1016/j.cnp.2017.09.003.

4. Wendling, F.; Ansari-Asl, K.; Bartolomei, F.; Senhadji, L. From EEG signals to brain connectivity: A model-based evaluation of 
interdependence measures. Journal of Neuroscience Methods 2009, 183, 9-18. doi:10.1016/j.jneumeth.2009.04.021. 5. Bashiri, M.; Mumtaz, W.; Malik, A.S.; Waqar, K. EEG-based brain connectivity analysis of working memory and attention. ISSBES 2015 - IEEE Student Symposium in Biomedical Engineering and Sciences: By the Student for the Student 2016, pp. 41-45.

doi:10.1109/ISSBES.2015.7435890.

6. Chang, S.; Dong, W.; Jun, H. Use of electroencephalogram and long short-term memory networks to recognize design pref erences of users toward architectural design alternatives. Journal of Computational Design and Engineering 2020, 7 , 551-562. doi:10.1093/jcde/qwaa045.

7. Krumpe, T.; Scharinger, C.; Rosenstiel, W.; Gerjets, P.; Spüler, M. Unity and diversity in working memory load: Evidence for the separability of the executive functions updating and inhibition using machine learning. bioRxiv 2018. doi:10.1101/389395. 8. Wu,

C.T.; Dillon, D.; Hsu, H.C.; Huang, S.; Barrick, E.; Liu, Y.H. Depression Detection Using Relative EEG Power Induced by Emo tionally Positive Images and a Conformal Kernel Support Vector Machine. Applied Sciences 2018, 8, 1244.

doi:10.3390/app8081244. 9. Kumar, P.; Garg, S.; Garg, A. Assessment of Anxiety, Depression and Stress using Machine Learning Models. Procedia Computer Science 2020, 171, 1989-1998. doi:10.1016/j.procs.2020.04.213.

10. Priya, A.; Garg, S.; Tigga, N.P. Predicting Anxiety, Depression and Stress in Modern Life using Machine Learning Algorithms. Procedia Computer Science 2020, 167, 1258-1267. doi:10.1016/j.procs.2020.03.442.

11. Hosseinifard, B.; Moradi, M.H.; Rostami, R. Classifying depression patients and normal subjects using machine learn ing techniques and nonlinear features from EEG signal. Computer Methods and Programs in Biomedicine 2013, 109, 339-345. doi:10.1016/j.cmpb.2012.10.008.

12. Schirrmeister, R.; Gemein, L.; Eggensperger, K.; Hutter, F.; Ball, T. Deep learning with convolutional neural networks for decoding and visualization of eeg pathology. arXiv 2017.

13. Johannesen, J.K.; Bi, J.; Jiang, R.; Kenney, J.G.; Chen, C.M.A. Machine learning identification of EEG features predicting working memory performance in schizophrenia and healthy adults. Neuropsychiatric Electrophysiology 2016, 2, 1-21. doi:10.1186/s40810- 016-0017-0.

14. Antonijevic, M.; Zivkovic, M.; Arsic, S.; Jevremovic, A. Using Al-Based Classification Techniques to Process EEG Data Collected during the Visual Short-Term Memory Assessment. Journal of Sensors 2020, 2020. doi:10.1155/2020/8767865. 15. Amin, H.U.;

Mumtaz, W.; Subhani, A.R.; Saad, M.N.M.; Malik, A.S. Classification of EEG signals based on pattern recognition approach.

Frontiers in Computational Neuroscience 2017, 11, 1-12. doi:10.3389/fncom.2017.00103.

16. Patel, M.J.; Khalaf, A.; Aizenstein, H.J. Studying depression using imaging and machine learning methods. Neurolmage: Clinical 2016, 10, 115-123. doi:10.1016/j.nicl.2015.11.003.

17. Sharma, A.; Verbeke, W.J.M.I. Improving Diagnosis of Depression With XGBOOST Machine Learning Model and a Large Biomarkers Dutch Dataset $(\mathrm{n}=11,081)$. Frontiers in Big Data 2020, 3, 1-11. doi:10.3389/fdata.2020.00015.

18. Julian, L.J. Measures of Anxiety. Arthritis Care 2011, 63, 1-11. doi:10.1002/acr.20561.Measures.

19. jcavanagh@unm.edu, J.F.C. "EEG: Probabilistic Selection and Depression", 2021. doi:10.18112/openneuro.ds003474.v1.1.0. 20.

Cavanagh, J.F.; Frank, M.J.; Broadway, J. "EEG: Visual Working Memory + Cabergoline Challenge", 2021.

doi:10.18112/openneuro.ds003519.21. Pavlov, Y.G.; Kotchoubey, B.; Pavlov, Y.G. Temporally distinct oscillatory codes of retention and manipulation of verbal working memory Corresponding author : 2021.

22. Pavlov, Y.G. "EEG: verbal working memory", 2021. doi:10.18112/openneuro.ds003565.v1.0.3.

23. Rubinov, M.; Sporns, O. Complex network measures of brain connectivity: Uses and interpretations. Neurolmage 2010, 52, 1059-1069. doi:10.1016/j.neuroimage.2009.10.003.

24. Gao, S.; Calhoun, V.D.; Sui, J. Machine learning in major depression: From classification to treatment outcome prediction. CNS Neuroscience and Therapeutics 2018, 24, 1037-1052. doi:10.1111/cns.13048.

25. Dhanapal, R.; Bhanu, D. Electroencephalogram classification using various artificial neural networks. Journal of Critical Reviews 2020, 7, 891-894. doi:10.31838/jcr.07.04.170.

26. Jiao, Z.; Gao, X.; Wang, Y.; Li, J.; Xu, H. Deep Convolutional Neural Networks for mental load classification based on EEG data. Pattern Recognition 2018, 76, 582-595. doi:10.1016/j.patcog.2017.12.002.

27. Kuanar, S.; Athitsos, V.; Pradhan, N.; Mishra, A.; Rao, K.R. Cognitive Analysis of Working Memory Load from Eeg, by a Deep Recurrent Neural Network. ICASSP, IEEE International Conference on Acoustics, Speech and Signal Processing Proceedings 2018, 2018-April, 2576-2580. doi:10.1109/ICASSP.2018.8462243.

28. Bilucaglia, M.; Duma, G.M.; Mento, G.; Semenzato, L.; Tressoldi, P. Applying machine learning EEG signal classification to emotion-related brain anticipatory activity. F1000Research 2020, 9, 173. doi:10.12688/f1000research.22202.1.

Version June 25, 2021 submitted to Journal Not Specified 12 of 12

29. Craik, A.; He, Y.; Contreras-Vidal, J.L. Deep learning for electroencephalogram (EEG) classification tasks: A review. Journal of Neural Engineering 2019, 16. doi:10.1088/1741-2552/ab0ab5.

30. Medvedev, A.V.; Agoureeva, G.I.; Murro, A.M. A Long Short-Term Memory neural network for the detection of epileptiform spikes and high frequency oscillations. Scientific Reports 2019, 9, 1-10. doi:10.1038/s41598-019-55861-w. 